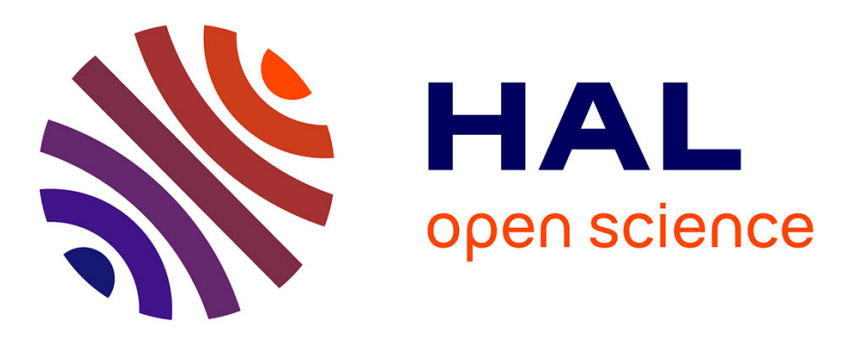

\title{
Structural modifications for squeal noise reduction: numerical and experimental validation
}

\author{
Francesco Massi, Laurent Baillet, Antonio Culla
}

\section{To cite this version:}

Francesco Massi, Laurent Baillet, Antonio Culla. Structural modifications for squeal noise reduction: numerical and experimental validation. International Journal of Vehicle Design, 2009, 51 (1-2), pp.168189. 10.1504/IJVD.2009.027120 . insu-00355110

\section{HAL Id: insu-00355110 https://hal-insu.archives-ouvertes.fr/insu-00355110}

Submitted on 22 Jan 2009

HAL is a multi-disciplinary open access archive for the deposit and dissemination of scientific research documents, whether they are published or not. The documents may come from teaching and research institutions in France or abroad, or from public or private research centers.
L'archive ouverte pluridisciplinaire HAL, est destinée au dépôt et à la diffusion de documents scientifiques de niveau recherche, publiés ou non, émanant des établissements d'enseignement et de recherche français ou étrangers, des laboratoires publics ou privés. 


\title{
Structural modifications for squeal noise reduction: numerical and experimental validation
}

\author{
Francesco Massi (corresponding author) \\ INSA-Lyon, LaMCoS, CNRS UMR5259 \\ F-69621, FRANCE \\ E-mail francesco.massi@insa-lyon.fr \\ Laurent Baillet \\ Laboratoire de Géophysique Interne et Tectonophysique, \\ CNRS, Université J. Fourier \\ BP 53, 38041 Grenoble cedex 9, FRANCE \\ E-mail laurent.baillet@ujf-grenoble.fr

\section{Antonio Culla} \\ University of Rome "La Sapienza" \\ Department of Mecanics and Aeronautics \\ Via Eudossiana 18, 00184 Rome, ITALY \\ E-mail antonio.culla@,uniroma1.it
}

\section{Keywords}

Squeal, contact, instability, lock-in, structural modification, numerical, experimental

\section{Abstract}

Squeal is an example of noise caused by vibrations induced by friction forces that lead to dynamic instability of the brake system. Several experimental, theoretical and numerical works have been carried out over the last six decades. Certain of these works have focused on simplified brake systems and identified 'lock-in' instability as being the cause of squeal. This paper presents a short bibliography of previous works and the most recent application of research developed using a simplified brake system called TriboBrake.

Commercial brake design is not capable of avoiding modal coupling due to 'lock-in' sensitivity to system dynamics, high modal density and uncertainties regarding the natural frequencies. This paper suggests an innovative approach for avoiding the growth of squeal vibrations, by making lumped structural modifications to the rotor. Experimental and numerical results are presented.

Biographical notes: Francesco Massi received his $\mathrm{PhD}$ degree from the Faculty of Mechanical Engineering of the University of Rome "La Sapienza" and from the doctoral school, MEGA at INSA, Lyon, in 2006. In 2007 he obtained the position of Maitre de Conferences at the Laboratory of Contact and Structural Mechanics, INSA - Lyon. His research activities focus on applied mechanics with emphasis given to vibrations, acoustics and tribological problems. His major topics are friction-induced vibrations, induced wear of contact surfaces under vibration, and the interaction between system dynamics and local behaviour at contact surfaces.

Biographical notes: Laurent Baillet received his $\mathrm{PhD}$ degrees from the doctoral school MEGA of the INSA of Lyon in 1994. Since 2005 he is Professor at the Joseph Fourier University. He is leader of the landslides team in the Laboratory of Geophysics and Tectonophysics. His areas of research include finite element formulation in large deformation frictional contact problem, dynamic rupture in frictional interface, rock-fall avalanche. 
Biographical notes: Antonio Culla, received is $\mathrm{PhD}$ in Theoretical and Applied Mechanics at the University of Rome "La Sapienza" in 2002 (dissertation: a confidence factor of statisticalenergetic solution of vibroacustic systems). His research topics are dynamics and vibroacoustic of autovehicles, naval vehicles, moored structures, offshore systems and launchers; his research interests includes control of vibration and noise techniques, statistical approach for analysing uncertain systems.

\section{1 - Introduction}

In spite of decades of investigation (Kinkaid et al., 2003), brake squeal is still an unresolved problem. Many attempts (Akay, 2002) have been made by industry and researchers to establish a general approach aimed at preventing squeal in brake design.

Different theories have been proposed to explain the self-excited vibrations that cause the emission of squeal sound: stick-slip instability, negative friction-velocity slope (Mills, 1938), sprag-slip mechanism (Spurr, 1961), local hammering (Chen et al., 2003), modal lockin (Akay et al., 2000), frictional follower forces (Mottershead, 1998). Kinkaid et al. (2003) wrote an extensive review of the main works available in the literature while Ouyang et al. (2005) recently presented a review of the finite element methods applied to squeal prediction and simulation.

Nowadays, the lock-in theory is one of the most accepted approaches for squeal generation and particular attention is given to the dynamics of brake systems. Moreover, one of the main difficulties encountered in studying squeal is the complexity of a real brake system. Thus many researchers approached the problem by conducting experimental and numerical analysis on simplified brake systems, and then trying to correlate the results with theoretical models.

The first to introduce the concept of modal coupling for explaining squeal instability was North (1972), who proposed a two-degrees-of-freedom model able to reproduce the unstable coupling by using an asymmetric stiffness matrix at the contact and a constant friction coefficient. Akay (2000) named the unstable coupling 'lock-in' after his investigations on a beam-on-disc set-up, one of the first simplified brake systems. The beamon-disc consists of a cantilever beam and a rotating disc pressed against each other using a dead weight. Similar systems were later used by Tuchinda et al. (2001) and Allgaier et al. (2002).

The same modal coupling mechanism was reproduced with the 'Laboratory Brake' (Giannini et al., 2006), with the third component of the brake system being introduced by adopting a brake pad of reduced dimensions. Experiments on this set-up allowed analysing the 'lock-in' between a mode of the pad and one mode of the disc. Moreover, by introducing a second brake pad, is possible to investigate high frequency squeal and the influence of pad dimensions (Giannini et al., 2008). Massi et al. (2005) obtained similar results by using real brake pads; these results make it possible to extrapolate the results obtained from simplified systems to real brakes.

The TriboBrake set-up (Massi et al., 2006a) is an evolution of the 'laboratory-brake'. It was designed to develop a parallel dynamic and tribological analysis to take into account the interdisciplinary aspects of the problem. Massi et al. (2006a) showed that it is possible generate squeal by coupling the disc dynamics with either the pad or the caliper dynamics. Clear identification of coupling mode instability was reported and two necessary conditions were highlighted (Massi et al., 2006a; 2007) in order to obtain unstable coalescence: i) closeness in frequency between the two coalescing modes; ii) high tangential and normal deformation of brake substructures (disc, pad and caliper) at the contact zone. Thus the oscillation of normal and friction forces at the contact surface allows unstable modal coupling. 
Massi et al. (2007) presented a numerical analysis for squeal prediction by using complex eigenvalue analysis; the values of the parameters that lead to instability are detected and introduced in a nonlinear finite element model developed with a code for explicit contact time simulation (Baillet and Sassi, 2002; Carpenter et al., 1991), to reproduce squeal vibrations. The nonlinearity of the contact is accounted for and the simulation results agree with the squeal behaviour encountered experimentally (Massi et al., 2006a). Moreover, the numerical simulations allowed calculating the behaviour of the local contact stresses during squeal, which are impossible to recover experimentally.

Tribological analysis (Massi et al., 2008a) of the contact surfaces after squeal allowed verifying the numerical results. Local oscillations of the contact stresses at the squeal frequency induce fatigue at the contact surface, leading to the occurrence of superficial material exfoliations and cracks.

The results obtained from tribological, dynamic investigations and simulations on the TriboBrake converge together, characterising squeal as a dynamic instability of the brake system due to unstable couplings between two modes of the brake components. Coupling occurs at the contact surface where the oscillations of the local contact stresses and the friction coefficient couple the normal and tangential vibrations of the brake components, causing self-excited vibrations of the system (Massi, 2006).

In a recent work, the uncertainties of the parameters affecting the system dynamics have been introduced into numerical and theoretical models for squeal prediction (Culla et al., 2007).

An experimental analysis developed on a 'beam-on-disc' set-up allowed for investigating the relationship between squeal propensity and modal damping (Massi et al., 2008b), highlighting the key role of the ratio between the modal damping of the two coupling modes; such results agree with theoretical and numerical investigations (Fritz et al., 2007; Duffour and Woodhouse, 2004).

Finally, the works on the simplified brake system allowed investigating and numerically reproducing squeal instability. Also dependence on several system parameters has been investigated. Nevertheless the conclusion of the authors, when accounting for the findings, is that it is not possible to design a 'squeal-free' brake system since the complexity of brake dynamics in the frequency range of interest, the uncertainties on system parameters due to industrial production, variable operating conditions (brake pressure, wear, external environment, etc.) and the different damping factors due to several surfaces in contact, do not allow for a reliable design capable of avoiding unstable coupling between system modes. This observation is the motivation for the present work, in which the authors propose an alternative approach to the problem.

\section{2 - Lumped rotor modifications}

The instability occurring in brake systems when two eigenfrequencies of the system coalesce and become unstable is known as mode lock-in (Akay et al., 2000): when changing the system parameters, two modes of the system can have similar frequencies and coalesce to make the system unstable. The two eigenvalues of the system keep the same frequency, but one of them reaches positive values of the real part, i.e. it is unstable (see Figure 12): therefore the system vibrations increase at the natural frequency of the unstable mode. The eigenvalues coalesce until the parameter variation makes them shift again to different frequencies and both the modes return to be stable (lock-out).

The main strategy adopted in brake design to predict and prevent squeal frequencies is parametrical complex eigenvalue analysis performed on the assembled system by FEM (Finite Element Modelling) (Ouyang et al., 2005; Cao et al., 2004; Massi et al., 2007). This approach consists in individuating the possible unstable coalescences and design modifications so as to 
either avoid the frequency coincidence between the two modes, or modify the geometrical coupling of the modes at the contact interface.

Nevertheless, commercial brakes are very complicated systems, characterised by several parts connected together and by high modal density. Moreover, due to mass production and variable operating conditions (wear, brake pressure, etc.), such systems suffer from high variability which is not easy to model. Because of these reasons, numerical eigenvalue analysis alone cannot lead to solving the problem.

Therefore, since it is impossible to produce brakes characterised by well defined dynamics in order to avoid coalescence, this paper proposes a different approach to the problem by using the introduction of lumped structural modifications on the disc. This allows continuous modification of the dynamics of the system thanks to disc rotation. The disc mode frequencies periodically shift back and forward between two values, depending on the position of the lumped modification with respect to the mode shapes. Therefore, if the mode of the disc is close in frequency to the other coalescing mode, they coalesce and separate periodically. The vibrations due to the lock-in between the pair of modes do not have enough time to increase due to repetitive look-out and they can be reduced until they completely disappear.

First the experimental investigation developed on the TriboBrake set-up is presented after which a theoretical and numerical analysis for predicting the instability and reduction of unstable coupling is presented.

\section{3 - The TriboBrake COLRIS}

\subsection{Experimental set-up}

The set-up, named TriboBrake COLRIS (COllaboration Lyon-Rome for Investigation on Squeal), consists of a rotating disc (the brake rotor) and a small friction pad pushed against the disc by weights positioned on the pad support used to represent the brake caliper (Figure 1).

\section{FIGURE 1}

Figure 1 - Experimental set-up TriBobrake COLRIS.

The disc is an automotive disc brake (internal diameter $140 \mathrm{~mm}$, external diameter 264 $\mathrm{mm}$, thickness $13 \mathrm{~mm}$ ) assembled on the shaft by two thick hubs that ensure the rigid behaviour of the connection. The brake pad is made of commercial friction material, obtained by machining standard brake pads. The support (central cylindrical body in the Figure 1) is also made of steel and its shape is chosen for its simple dynamics. The normal force between pad and disc (braking pressure) can be varied, by adding weights from 25 and $225 \mathrm{~N}$ on the top of the support (average contact pressure from 0.25 to $2.25 \mathrm{MPa}$ ). Two thin plates hold the pad support in the tangential direction. This solution permits obtaining low (negligible in non deformed vertical condition) stiffness in the normal direction and high stiffness in the tangential direction, which is necessary to oppose the friction force. A complete description of the TriboBrake along with the experiments carried out to characterise the squeal behaviour is presented in (Massi et al., 2006a).

\subsection{Set-up dynamics}

Because of its key role in the occurrence of squeal, the dynamics of the set-up is identified and monitored during the experimental tests. A preliminary EMA (Experimental Modal Analysis) is performed on the assembled system, i.e. when the pad is in contact with the disc. The analysis leads to distinguishing three different substructures: disc, support and pad. The dynamics of the assembly can be analysed as the sum of the disc, pad, and support 
dynamics. In fact the reduced contact surface $(10 \mathrm{~mm} \times 10 \mathrm{~mm})$ between the pad and the disc allows low coupling between the tangential dynamics of the pad (or support) and the normal (bending) dynamics of the disc. Therefore, when referring to the whole system, the following notation is used: the modes of the coupled system that involve bending vibration of the disc are called "disc modes", since the greater part of the energy is concentrated on the disc. Also, the modes involving the bending vibration of the pad (or support) are named "pad (or support) modes". When the modes draw close in frequency, both substructures vibrate (Massi et al., 2006a).

In particular, attention is focused on three sets of system modes that are considered to be the cause (Massi et al., 2006a; 2007) of the squeal instability of the set-up: the bending modes of the disc (normal direction with respect to the friction surface), the bending modes of the support (tangential direction with respect to the friction surface) and the tangential modes of the friction pad.

The bending modes of the disc are characterised by nodal diameters and nodal circumferences: the $(\mathrm{n}, \mathrm{m})$ mode of the disc is characterised by n nodal circumferences and $\mathrm{m}$ nodal diameters. The disc is characterised by an axial symmetry: therefore the modes of the disc are generally double modes. When placed in contact with the pads, the disc looses its axial symmetry, so that the modes of the disc are no longer double modes and they split (Massi et al., 2006a).

The following notation is used to denote the split modes (Table 1):

- $\quad$ mode $(\mathrm{n}, \mathrm{m}-)$ a nodal diameter is coincident with the contact point;

- $\quad$ mode $(n, m+)$ an anti-node is coincident with the contact point.

The support modes are analysed by an SIMO (Single-Input-Multi-Output) analysis, exciting the support in the tangential direction close to the contact area. Five tangential modes of the support are recognised in the frequency range of interest (Massi et al., 2006a). Only the second and the third modes of the support are characterised by the largest tangential displacement at the contact area and, consequently, they are the only modes of the support involved in squeal instability (Massi et al., 2006a). The second mode is a rigid rotational mode of the support, while the third mode is the first bending mode of the support (II and III support modes in Table 1).

The friction pad is the third substructure to be investigated; its dynamics is easily recognisable in the assembled dynamics. Figure 2 shows the PSD (Power Spectral Density) of the pad acceleration in the tangential direction during brake tests without squeal. The first three peaks in frequency correspond to three support modes. The others two peaks at $4 \mathrm{kHz}$ and $11.1 \mathrm{kHz}$ correspond to the modes of the pad (I and II in Table 1).

\section{FIGURE 2}

Figure 2 - PSD of the tangential acceleration of the pad during braking phase without squeal.

During experiments, in order to achieve the coincidence in frequency between two appropriate modes of the system, the variation of the normal load is used to modulate its dynamics. The ranges covered by each mode are reported in table 1 .

\section{TABLE 1}

Table 1 - Ranges of frequency covered by the system modes. Frequency range corresponds to the variation of the normal load. 


\section{4 - Model of the set-up}

\subsection{Linear finite element model}

The geometry of the FE (Finite Element) models of the TriboBrake is reported in Fig. 3. ANSYS ${ }^{\circledR}$, commercial FE software, is used to investigate the dynamics of the brake system and to calculate its complex eigenvalues as a function of the system parameters. The element SOLID45 (3D brick element with linear shape functions) is adopted to mesh all the solid components of the system. The brake disc is clumped at the internal circumference, where the hubs hold it into the experimental set-up. The brake pad consists of a cube $(10 \mathrm{~mm} \times 10 \mathrm{~mm} \times 10 \mathrm{~mm})$. The pad support is modelled by a beam $(10 \mathrm{~mm} \times 10 \mathrm{~mm} \times 100 \mathrm{~mm})$ and a thin layer $(5 \mathrm{~mm})$ of high density is attached to the top of the support and simulates the mass added by the weights placed on the experimental set-up. Four rows of springs, two on each side, hold the beam in the horizontal (friction force) direction, and model the thin aluminium plates that hold the pad support in the experimental set-up.

\section{FIGURE 3}

\section{Figure 3. Finite element model of the experimental set-up.}

To introduce the sliding contact between disc and pad the contact elements CONTA173 and the target elements TARGE170 have been introduced at the pad and disc contact surfaces respectively. A 'surface to surface contact' model of ANSYS is adopted. A prestressed modal analysis is performed to account for the system deformation and the contact distribution into the stationary sliding condition. The load at the top of the support is applied in an initial load step after which the rotation of the disc is imposed and, finally, a modal analysis on the deformed system is performed.

\subsection{The substructured model}

Because of the large number of solutions needed to perform the parametrical analysis and because of the considerable computational effort necessary to obtain each solution of the asymmetric problem, the FE model has been substructured. Substructuring is a procedure that condenses a group of finite elements into one element represented as a matrix. The substructure routine of ANSYS is used.

Three main substructures of the brake system are considered here: the support (caliper), the pad and the disc. The single-matrix element is referred to as a super-element. Each super-element is made of the same elements and nodes as in the complete model; moreover, the master nodes connect the super-element to the other parts of the model and approximate the eigenvectors of the corresponding substructure.

The disc is characterised by a mapped mesh of 12600 elements. The super-element representing the support includes the beam and the mass at the top ( 426 elements). The pad is modelled by 1725 brick elements. The substructures are then connected and constrained in the final model. In particular, the same contact elements described above are used to reproduce the contact. A preliminary parametrical eigenvalue analysis was performed with the complete and substructured models: the percentage error on the eigenvalues of interest is less then $2 \%$.

\section{5 - Experimental results}

\subsection{System dynamic modification}

The tests reported below highlight the effect of lumped structural modifications introduced into the disc on the system dynamics. In particular the addition of a lumped mass 
is analysed here. The lumped mass is attached with a layer of wax at the disc periphery. The idea is to introduce an asymmetry that changes continuously position respect to the disc modes, varying continuously the system dynamics as reported below.

Two asymmetries are then introduced regarding the axial-symmetric structure of the disc: the contact with the friction pad and the lumped mass. During the braking phase the former is fixed in space; on the contrary, the mass rotates with the disc.

The authors showed in (Massi et al., 2006a) that the $\left(n, m^{-}\right)$and $(n, m+)$ modes of the disc are fixed in space having a node and an anti-node of vibration respectively at the contact point with the pad. While the disc rotates, the relative position between the two asymmetries (pad and mass) changes. Because of the larger effect due to the contact with the pad, the modes stay fixed with the stationary frame, while the mass rotates with the disc. When referring to the position of the lumped modification (on the outer circumference of the disc) with respect to the disc (stationary) modes, the following notation is used in this paper: position $\mathbf{A}$ is the node of vibration of the mode $(n, m+)$, and anti-node of vibration of the mode ( $n, m-)$; position $\mathbf{C}$ is the anti-node of vibration of the mode $(n, m+)$, and node of vibration of the mode (n,m-); position $\mathbf{B}$ is between $\mathbf{A}$ and $\mathbf{C}$. The closer the lumped modification is to the anti-node of vibration, the more it affects the mode frequency. Consequently the lumped modification affects the frequency of mode $(n, m+)$ when it is at the position $\mathbf{C}$; it affects the frequency of mode $(n, m-)$ when it is at the position A. Due to the low value of the modification the modes of the disc are slightly modified by its introduction; this is why in the following they are considered to be fixed with the stationary frame (pad) and the analysis is focused only on the shift of the natural frequencies.

Figure 4-a shows the respective FRFs (Frequency Response Functions) measured for the three different positions of the mass $(\mathbf{A}, \mathbf{B}$ and $\mathbf{C})$ with respect to mode $(0,4)$. Figure 4-b shows the FRFs for the three different positions with respect to mode $(0,7)$. When the lumped mass (10 gr) passes through a node of the mode $(0,4+)$ (position $\mathbf{A}$ in Figure 4-a) its natural frequency is at $3250 \mathrm{~Hz}$, which is equal to its frequency without the mass. If the mass moves toward its anti-node of vibration (position $\mathbf{C}$ ) the natural frequency decreases to $3220 \mathrm{~Hz}$. The greater the deformation at the point where the mass is added, the more the frequency decreases. The same effect is visible for mode $(0,4-)$ passing from position $\mathbf{C}$ to position $\mathbf{A}$.

On the contrary, Figure 4-b shows that mode $(0,7+)$ increases in frequency (from 8730 to $8760 \mathrm{~Hz}$ ) when the lumped modification is at its anti-node of vibration (position $\mathbf{C}$ in Figure 4-b). This behaviour can be explained by the fact that the mass is attached by a layer of wax and it has finite dimensions: thus the modification cannot be considered just as a lumped mass. The behaviour of the mode frequencies of the set-up respect to the addition of the mass is function of the mode order (frequency) and it is repeatable; therefore it is used for the tests

\section{FIGURE 4}

Figure 4: FRFs for different positions of the mass $(C$ is the anti-node of vibration of the modes $(n, m+)$, A its node of vibration); the arrows indicate the shift of the mode frequencies when the mass passes from position $A$ to position $C$.

Figure 5 shows the behaviour of the frequencies of modes $(0,4+)$ and $(0,7+)$ as a function of the value of the added mass when the mass is added at their anti-node of vibration (position C). The effect of the modification is clear: the greater the mass, the greater the expected shift in frequency.

FIGURE 5

Figure 5: FRFs for different values of the mass in position $C$ (anti-node of vibration of modes $(n, m+)$; the arrows indicate the shift of mode frequencies with increasing mass. 
During the braking phase the lumped mass moves $2 m$ times from a node to an antinode of the $(n, m+)$ mode and vice versa, at each complete rotation of the disc. Thus the natural frequency of the $(n, m+)$ mode shifts backwards and forwards with an oscillation period equal to $2 m$ times the period of the disc rotation.

The lumped structural modification thus allows continuously modifying the disc dynamics, oscillating all the natural frequencies of the disc bending modes between two extreme values.

\subsection{Squeal reduction by the addition of mass}

Once the system parameters (normal load equal to $44 \mathrm{~N}$ ) are set for the frequency coincidence between the second pad mode and the $(0,7+)$ mode, squeal occurs at $8730 \mathrm{~Hz}$ during every braking phase. A harmonic acoustic emission over $100 \mathrm{~dB}$ is recognised.

The lumped mass is then attached to the periphery of the disc. In view to not introducing eccentricity on the disc, the added mass is split into two equal parts that are attached diametrically at the disc periphery. The effect on the bending modes of the disc is equal, due to the axial symmetry of the modes.

During the braking phase, the tangential (friction direction) velocity of the pad is measured by a laser vibrometer. Figure 6-a presents the squeal vibrations measured during the braking phase with an added mass of 5 gr; at 12 seconds the mass is suddenly removed, and the squeal is continuous. Because of the sensitivity of squeal to the system parameters, all the measurements presented below are performed into the same way: the mass is detached during the braking phase at the end of each measurement, to make sure that comparable squeal conditions, characterised by the same frequency and vibration amplitude, are obtained. Figure 6-a shows a periodic increase $(8745 \mathrm{~Hz})$ and decrease $(8730 \mathrm{~Hz})$ of the squeal vibrations until the mass is detached: the squeal vibrations are significantly reduced.

Lock-in/lock-out periodicity $(T d / 2 m)$ is visible in Figure 6-b, which shows the normalised spectrogram of the velocity of the pad during squeal. The spectrogram in Figure 6$\mathrm{b}$ is normalized to the value of the maximum peak in frequency at each time interval, in order to clearly track the vibration frequency of the system. The vibration frequency follows the frequency oscillation of mode $(0,7+)$ between the two extreme values (Figure 4-b and Table 2). This oscillation is due to the rotation of the mass with the disc: the mass passes periodically by vibration anti-node and node of mode $(0,7+)$. The system dynamics is characterised by successive lock-in and lock-out configurations. When the disc natural frequency draws close to the pad natural frequency $(8730 \mathrm{~Hz})$, the squeal vibrations increase (lock-in); while they decrease when the disc natural frequency moves to larger $(8745 \mathrm{~Hz})$ frequencies (lock-out). The period of the lock-in occurrence is equal to the disc rotation period $T d$ ratio twice the number of nodal diameters $(2 \mathrm{~m})$. Figure 6-c shows the spectrogram of the non-normalized velocity, to highlight the decrease of squeal vibrations during the lockout periods. The vibrations are almost eliminated. As soon as the mass is removed (12 s) the squeal vibrations return to the original amplitude at a constant frequency.

\section{FIGURE 6}

Figure 6: Tangential velocity of the pad (a), normalized spectrogram (b) and classical spectrogram (c) when a mass of $5 \mathrm{gr}$ is attached at the disc periphery; the mass is removed suddenly at $12 \mathrm{~s}$ and the squeal vibrations increase again. Unstable coupling between the II pad mode and the mode $(0,7+)$ with disc velocity of $15 \mathrm{rpm}$. Td is the period of rotation of the disc.

Table 2 shows the frequency interval as a function of the added mass in position $\mathbf{C}$. By increasing the added mass, the frequency interval covered by the $(0,7+)$ mode, during disc 
rotation, increases. The natural frequency of the (0,7-) mode is not affected by the mass, which falls at its node of vibration.

\section{TABLE 2}

Table 2: Frequency ranges covered by the $(0,7+)$ mode.

Because the period of variation is fixed with the number of the nodal diameters and the disc velocity, the velocity of the frequency shift increases with the wideness of the interval covered (Table 2).

Figure 7 shows the tangential velocity measured at the pad side during the braking phase when different values of the added mass are used. The black plot is pad acceleration during braking phase when $1 \mathrm{gr}$ is attached to the disc periphery. A low modulation of the squeal amplitude is recognised, but the mass is too light to significantly affect the vibrations. The blue and the green plots are the pad velocity during braking phase when 2.5 and 5 gr respectively are attached to the disc periphery. In this case the frequency range covered by the natural frequency of the disc is larger and the squeal vibrations increase and decrease periodically, with a periodicity equal to 14 times ( $2 m$ times) the period of the disc rotation. When increasing the added mass, the disc natural frequency moves faster (same period but larger interval of the frequency shift), and the squeal vibrations do not have enough time to increase (the lock-in configuration is maintained for a shorter time). Figure 7 shows that squeal vibrations are reduced by increasing the mass. Finally, the red plot shows the pad velocity when the added mass is equal to $10 \mathrm{gr}$ and the squeal vibrations are completely eliminated.

Again, to verify the continuity of squeal the mass is detached (after 6.5 seconds for the measure with 10gr in Figure 7), and squeal restarts at its original amplitude and frequency.

\section{FIGURE 7}

Figure 7: Tangential velocity of the pad when 1gr (black), 2.5gr (blue), 5gr (green) and 10gr are attached to the disc periphery. The mass is detached after 6.5 seconds for the measurement with $10 \mathrm{gr}$. Unstable coupling between the II pad mode and the mode $(0,7+)$.

\subsection{Effect of disc velocity}

The effect of increasing the value of the mass is to increase the frequency range covered by the disc mode during the same period and consequently to reduce the time for lock-in. Another way to reduce time for increasing vibrations during lock-in (with lumped modifications) is to increase the disc rotational velocity, i.e. to decrease the period of the frequency oscillation $(T d / 2 m)$.

Figure 8 -a shows the pad velocity during squeal when a mass of $5 \mathrm{gr}$ is added to the disc and the disc velocity is increased from $6 \mathrm{rpm}$ (DISC VELOCITY A) to 12rpm (DISC VELOCITY B) and finally to 50rpm (DISC VELOCITY C). The figure shows the reduction of the lock-in /lock-out period when passing from velocity A to velocity B; moreover a reduction of the squeal vibration amplitude is observed. When the disc rotates at the higher velocity the squeal emission almost disappears and the pad velocity gets lower (Figure 8-a, $21 \mathrm{~s}<\mathrm{t}<27 \mathrm{~s}$ ). When the mass is detached (the arrows in figure 8) the squeal vibration return to the original amplitude and frequency.

Figure 8-b shows the same test with an added mass of $7.5 \mathrm{gr}$; the same behaviour is highlighted, but for the disc velocity value $\mathrm{C}$, squeal disappears completely (Figure 8-b, $13 \mathrm{~s}<\mathrm{t}<20 \mathrm{~s}$ ). In fact, in this case the time for lock-in and for vibration to increase is reduced by both increasing the disc velocity and increasing the amplitude of the frequency shift. 
It is opinion of the authors that this behaviour is the reasons why automotive squeal only occurs at low velocity. Numerically (Baillet et al., 2006) and experimentally (Giannini et al., 2006) it has been shown that squeal amplitude increases linearly with disc velocity up to a maximum value and then stays constant when disc velocity increases. In fact, the simplified set-ups are designed to have constant boundary conditions, i.e. constant dynamics during the braking phase. Real brakes under real operational conditions are characterised by non constant dynamics; in particular the imperfect balancing of the brake disc allows continuously changing lock-in conditions during disc rotation. Therefore, the absence of squeal for high velocity could be due to the quick shift of the system's natural frequencies and thus to the less time in which the vibration can increase.

\section{FIGURE 8}

Figure 8: Tangential pad velocity for three different values of the disc rotational velocity $(A=6 \mathrm{rpm}, \mathrm{B}=12 \mathrm{rpm} ; \mathrm{C}=50 \mathrm{rpm})$, when masses of $5 \mathrm{gr}$ (a) and 7.5gr (b) are added to the disc periphery. The arrows indicate when the mass is detached.

\subsection{Effect of mode order}

The results presented here for squeal events due to coalescence between the II pad mode and the $(0,7+)$ mode (squeal at $8730 \mathrm{~Hz}$ ), were also obtained for the other squeal frequencies that involve disc bending modes. Figure 9 shows the squeal vibrations when a mass of 5gr is added to the disc periphery. The black plot is the pad velocity for the case presented above when the $(0,7+)$ mode couples with the pad mode. The grey plot is the support tangential acceleration measured during squeal when the $(0,4+)$ mode of the disc couples with a bending mode of the support at $3.24 \mathrm{kHz}$. The effect of the addition of the mass is the same, while the period of the lock-in / lock-out cycle changes with the mode order $m$, as shown in Figure 9. The comparison between the squeal amplitudes cannot be performed because of the completely different coupling conditions.

\section{FIGURE 9}

Figure 9: Pad velocity for squeal at $8.7 \mathrm{kHz}$ and $5 \mathrm{gr}$ of added mass (black); support tangential acceleration (scaled) for squeal at $3.25 \mathrm{kHz}$ and 5 gr of added mass.

\section{6 - Numerical results}

\subsection{Theoretical analysis}

For lumped modifications the problem related to both the prediction and optimization aspects was completely solved. The prediction problem allows estimating the response of the new system once the dynamics of the original system and the dynamics of the modifications are known (Sestieri and D'Ambrogio, 1972; Rivin and D'Ambrogio, 1990; D'Ambrogio and Sestieri, 2001). The inverse (optimisation) problem allows calculating the modification that brings about the desired dynamic behaviour of the system.

As the original structure is known by its modal data (natural frequencies and mode shapes) or by its FRF matrix measured on a set of points, the relationships describing the dynamics of the new modified system can be written.

The original structure has the following classical equation of motion:

$$
\mathbf{M} \underline{\ddot{x}}_{0}+\mathbf{C} \underline{\dot{x}}_{0}+\mathbf{K} \underline{x}_{0}=\underline{f}
$$

Where $\underline{x}_{0}$ is the coordinate vector, $f$ is the forces vector, $\mathbf{M}$ and $\mathbf{K}$ are the mass and stiffness matrices, and the proportional model for the damping of the structure is assumed: $\mathbf{C}=\alpha \mathbf{M}+\beta \mathbf{K}$. 
Let $\Phi$ and $\Lambda$ be the eigenvectors and the eigenvalues matrices of the undamped original structure, respectively, $\underline{q}_{0}$ the modal coordinate vector and $\tilde{f}$ the modal forces vector. Equation (1) can be rewritten in the modal frame as follows:

$$
\mathbf{I} \underline{\ddot{q}}_{0}+(\alpha \mathbf{I}+\beta \boldsymbol{\Lambda}) \underline{\dot{q}}_{0}+\Lambda \underline{q}_{0}=\tilde{f}
$$

Let $\Delta \mathbf{M}$ and $\Delta \mathbf{K}$ be the mass and the stiffness matrices of the modifications. The modified structure equation of motion has the following formal shape:

$$
(\mathbf{M}+\Delta \mathbf{M}) \underline{\ddot{x}}+[\alpha(\mathbf{M}+\Delta \mathbf{M})+\beta(\mathbf{K}+\Delta \mathbf{K})] \underline{\dot{x}}+(\mathbf{K}+\Delta \mathbf{K}) \underline{x}=\underline{f}
$$

which in modal coordinates is:

$$
\left(\mathbf{I}+\boldsymbol{\Phi}^{T} \Delta \mathbf{M} \boldsymbol{\Phi}\right) \underline{\ddot{q}}+\left[\alpha\left(\mathbf{I}+\boldsymbol{\Phi}^{T} \Delta \mathbf{M} \boldsymbol{\Phi}\right)+\beta\left(\Lambda+\boldsymbol{\Phi}^{T} \Delta \mathbf{K} \boldsymbol{\Phi}\right)\right] \underline{\dot{q}}+\left(\Lambda+\boldsymbol{\Phi}^{T} \Delta \mathbf{K} \boldsymbol{\Phi}\right) \underline{q}=\underline{\tilde{f}}
$$

By solving the new eigenproblem, a new set of mode parameters are calculated: $\boldsymbol{\Phi}_{N}$ and $\boldsymbol{\Lambda}_{N}$. Equation (4) can be rewritten as follows:

$$
\underline{\ddot{q}}_{N}+\left(\alpha \mathbf{I}+\beta \boldsymbol{\Lambda}_{N}\right) \underline{\dot{q}}_{N}+\boldsymbol{\Lambda}_{N} \underline{q}_{N}=\boldsymbol{\Phi}_{N}^{T} \underline{\tilde{f}}
$$

The natural frequencies of the modified structure can be calculated by $\boldsymbol{\Lambda}_{N}$ and the eigenvectors of the new structures are: $\boldsymbol{\Psi}=\boldsymbol{\Phi} \boldsymbol{\Phi}_{N}$ (Sestieri and D'Ambrogio, 1972). In fact, since $\underline{x}$ is the vector of the physical coordinates then: $\underline{x}=\boldsymbol{\Psi} \underline{q}_{N}$.

In general the mass and stiffness matrices of the system are not known and the dynamics of the structure is known by the FRF matrix achieved by measurements. Let $\mathbf{H}$ be the FRF matrix of the original system, i.e. $\underline{x}=\mathbf{H} \underline{f}$, and $\Delta \mathbf{B}$ the matrix of the modifications (D'Ambrogio and Sestieri, 2001), i.e. $\Delta \mathbf{B}=-\omega^{2} \Delta \mathbf{M}+j \omega(\alpha \Delta \mathbf{M}+\beta \Delta \mathbf{K})+\Delta \mathbf{K}$.

Let $\left(\mathbf{H}^{-1}+\Delta \mathbf{B}\right) \underline{x}_{N}=\underline{f}$ the formal relationship of the modified structure where $\underline{x}_{N}$ is the coordinate vector of the new system. $\Delta \mathbf{B}$ and $\mathbf{H}^{-1}$ are calculated at the same degrees of freedoms. Therefore, the new FRF matrix is $\mathbf{H}_{N}=\left(\mathbf{H}^{-1}+\Delta \mathbf{B}\right)^{-1}$. By performing algebraic calculations it is possible to calculate the FRF matrix of the new modified structure by reducing the number of inversions (D'Ambrogio and Sestieri, 2001), thus the following relationship is adopted:

$$
\mathbf{H}_{N}=(\mathbf{I}+\mathbf{H} \Delta \mathbf{B})^{-1} \mathbf{H}
$$

Relationships (5) and (6) allow solving the direct problem, in other words they give the dynamics of the modified structure (mode parameters or FRF matrix) by providing knowledge of the original system and of the dynamics modifications.

The goal of the case studied in this paper is the shift of the resonances of the $(n, m+)$ modes in order to avoid lock-in. One way of achieving this is to consider a lumped structural modification and verify the efficiency of the modification by Equation (3). To reproduce the effect of the added mass in the experimental tests presented above a lumped modification corresponding to a mass-damper-spring system is introduced. Indeed since the mass added experimentally is attached to the disc by a layer of wax for accelerometers and has finite dimensions, it would be not correct to model it by a lumped mass.

A set of measurements are performed on the disc and a matrix of FRF of the unmodified structure can be considered. Since the modification involves only one point of the structure, the modification matrix $\Delta \mathbf{B}$ is zero everywhere except for the element corresponding to the position of the lumped mass-damping-spring system. Moreover, the drive point is at the position selected for adding the one degree of freedom system. So, by 
calling the drive point 1, a simple relationship can be calculated for element $H_{N 11}$ of the new system FRF matrix:

$$
H_{N 11}=\left(1+H_{11}\left(m-j \frac{c}{\omega}-\frac{k}{\omega^{2}}\right)\right)^{-1} \cdot H_{11}
$$

where $m, c, k$ are the coefficients of the lumped mass-damper-spring.

In Figures 10 and Figure 11 the original FRFs measured on the experimental set-up without added mass are plotted in black; the corresponding FRFs of the modified system calculated by Equation (7) are shown in grey. Figure 10a and 10b show the FRFs measured at position $\mathbf{C}$ (anti-node of vibration) of modes $(0,4+)$ and $(0,7+)$ respectively. As expected from the experimental tests (Figure 5) mode $(0,4+)$ decreases in frequency while mode $(0,7+)$ increases in frequency (by about $30 \mathrm{~Hz}$ with a mass of $10 \mathrm{gr}$ ). Figure 11 shows the measured (black) and modified (grey) FRFs when the modification is added at position $\mathbf{B}$ of mode $(0,4)$. In this case the measured FRFs show both modes $(0,4+)$ and $(0,4-)$; the introduction of the modification at position $\mathrm{B}$ allows shifting both mode frequencies, as found by the tests (Figure 4a). When introducing the lumped modification, the amplitude of the response of the mode also decreases, as measured experimentally (Figure 4).

\section{FIGURE 10}

Figure 10: Comparison between the FRFs of the original system (experimental) and the modified structure by the addition of a lumped mass-damping-spring modification (mass of $10 \mathrm{gr})$. The modification is introduced at the anti-node of modes $(n, m+)$.

\section{FIGURE 11}

Figure 11: Comparison between the FRFs of the original system (experimental) and the modified structure by the introduction of the lumped mass-damping-spring modification (mass of $10 \mathrm{gr}$ ) at position $B$ of mode $(0,4)$.

The goal of the case studied in this paper is to shift the resonance of the disc in order to avoid lock-in. The prediction problem solved above gives the same behaviour as that obtained by the tests. The inverse problem can be reformulated to optimize the structural modifications in order to obtain the desired frequency shift of the rotor modes. This theoretical method for analysing and predicting the effect of lumped modification is particularly useful because it can be formulated for both the experimental (FRFs) and numerical (eigenvectors) data of the brake system.

\subsection{Complex eigenvalue analysis}

The complex eigenvalue analysis provides the tool for tracing the instability regions of the system. The numerical eigenvalue extraction is performed with the QRDamped Method by ANSYS and repeated as a function of the driving parameters. In (Massi et al., 2007) the Young's modulus of the pad material and the stiffness of the springs that hold the support have been chosen as the driving parameters that allow reproducing the dynamic behaviour of the experimental set-up as a function of normal load. Thus the extraction of the eigenvalues of the system is performed as a function of these two parameters to perform the parametrical analysis and identify squeal instabilities. Squeal is characterised by lock-in (Figure 12-a) between two eigenvalues of the system that start to have the same imaginary part (frequency), though they have opposing real parts with respect to the starting value (structural damping). Also, one of them succeeds in having a positive real part (Figure 12-b), i.e. it is unstable. A complete parametrical analysis for squeal prediction between 1 and $20 \mathrm{kHz}$ has been 
presented in (Massi et al., 2007) and (Massi et al., 2006c). In this section instability due to the coupling (lock-in) between mode $(0,4+)$ and the third tangential mode of the support is analysed. The same lock-in has been obtained experimentally and gives the origin of the squeal at $3240 \mathrm{~Hz}$ (Figure 9). In figure 12, the mode of the support increases in frequency until reaching mode $(0,4+)$, when they couple and one of the modes become unstable. On the contrary, mode $(0,4-)$ stays constant in frequency because contact with the pad is at its nodal diameter and it is not affected by the variation of the parameters chosen. The friction coefficient adopted for the analysis is equal to 0.4. A detailed analysis of the relationship between the friction coefficient and squeal prediction is reported in (Massi et al., 2007).

\section{FIGURE 12}

Figure 12: Lock-in between mode $(0,4+)$ and the mode of the support: a) eigenvalues as a function of the system parameters; the black dot are the eigenvalues with a positive real part (unstable); b) in the locus plot one of the two modes reaches the positive real semi-plane and becomes unstable.

The effect of a lumped modification is investigated here by adding a lumped mass to the periphery of the model. In particular, to reproduce the modification used in the experimental set-up an element with dimensions $(5 \mathrm{~mm} \times 5 \mathrm{~mm} \times 5 \mathrm{~mm})$ is attached to the periphery of the disc and its density is modified to perform a sensitivity analysis of the complex eigenvalues and thus of squeal lock-in.

The analysis has been performed for three positions of the mass with respect to mode $(0,4+)$ : position $\mathbf{A}$ falls at the vibration node of mode $(0,4+)$; position $\mathbf{C}$ falls at the anti-node of vibration of mode $(0,4+)$; position $\mathbf{B}$ is in between $\mathbf{A}$ and $\mathbf{C}$ (Figure $4-a)$.

Figure 13 shows the behaviour of the eigenvalues when varying the value of the added mass and the other system parameters are fixed to have instability $(\mathrm{E}=4200 \mathrm{MPa}$ and $\mathrm{k}=3.85$ $10^{7} \mathrm{~N} / \mathrm{m}$ in Figure 12). When no mass is added, the system is unstable at $3410 \mathrm{~Hz}$, where mode $(0,4+)$ couples with the support modes. Mode $(0,4-)$ is at $3340 \mathrm{~Hz}$.

In Figure 13-a the mass is added at position $\mathbf{C}$, mode $(0,4+)$ is affected by the mass and it shifts to lower frequencies. The system became stable because of lock-out. This is what occurs when the mass passes by the anti-node of vibration and squeal vibrations decrease (Figure 9); the greater the mass, the greater the distance in frequency (Figure 13) between the unstable couple of modes.

In Figure 13-c the mass is added at position $\mathbf{A}$, mode (0,4-) shifts to lower frequencies and mode $(0,4+)$ stays at the frequency of coincidence with the support mode. The system is unstable, independently of the value of the mass. This is what occurs when the mass passes by the node of vibration and the squeal vibrations increase (Figure 9);

Figure 14 shows the same analysis when the system parameters are fixed to not have instability $\left(E=4010 \mathrm{MPa}\right.$ and $\mathrm{k}=3.6610^{7} \mathrm{~N} / \mathrm{m}$ in Figure 12$)$. The system is stable without the added mass. By increasing the added mass in position $\mathbf{C}$, mode $(0,4+)$ decreases in frequency until it reaches the support mode and the instability appears. In fact, the aim of the lumped modification is not to avoid unstable coupling, but to periodically modify the system dynamics to avoid continuous lock-in and thus avoid increasing the squeal vibrations.

\section{FIGURE 13}

Figure 13: Effect of the addition of mass on the eigenvalues when the system parameters are set to obtain squeal without mass: a) when the mass is added in position $\mathrm{C}$ (anti-node of mode $(0,4+))$; b) the mass is added in position B; c) the mass is added in position A. In this last case squeal is not affected because the mass is at the node of mode $(0,4+)$. 


\section{FIGURE 14}

Figure 14: Effect of the addition of mass on the eigenvalues when the system parameters are set to obtain no squeal without mass (First solution on the $x$ axis of Figure 12): a) the mass is added in position $\mathrm{C}$; b) the mass is added in position $\mathrm{B}$; $\mathrm{c}$ ) the mass is added in position $\mathrm{A}$.

\section{Concluding remarks}

Nowadays, complex modal analysis for squeal prediction is one of the most commonly adopted approaches for reducing unstable conditions. Nevertheless, the high modal density characterising commercial brakes and the uncertainties on their dynamics due to mass production, do not allow developing a predictive tool for brake design without squeal.

By the understanding of the lock-in instability, the approach presented in this paper aims to eliminate squeal vibrations by introducing a structural modification in the rotor. The goal is to prevent vibrations from increasing at different squeal frequencies (all the squeals that involve bending modes of the disc) by a simple and inexpensive solution. In fact, the asymmetry introduced in the rotor (the added mass in this paper) makes the natural frequencies of the disc bending modes shift backwards and forwards, thus with continuous lock-in and lock-out configurations. Experimental tests show that by introducing a large enough modification it is possible to completely eliminate squeal vibrations.

The period of mode frequency oscillations is a function of disc rotational velocity and the number of nodal diameters. The amplitude of frequency oscillation is a function of the value of the lumped modification. It has been shown that increasing either the disc velocity or the value of the modification allows reducing the time for squeal vibrations to increase (reduced time for lock-in), and permits the elimination of squeal. The absence of squeal in real brakes at high velocity can be attributed to quick variations of system dynamics, due to the non constant dynamics with the disc rotation, which prevents vibration from increasing. This can explain why squeal occurs only at low rotational velocity.

Any lumped modification can be used to achieve the same results as those presented above. For instance, a well-arranged distribution of disc perforations, usually introduced to reduce heating, could be designed. Particular attention should be given to the distribution of the lumped modifications, in order to avoid the introduction of eccentricity in the disc, and to correctly assigning all the bending modes assumed to be involved in squeal.

A further investigation is scheduled to recover the optimal distribution of the modifications and to reproduce by a nonlinear numerical model the squeal suppression with lumped modifications. 


\section{References}

Akay A. (2002) 'Acoustic of friction', Journal of the Acoustical Society of America, Vol. 111(4), pp. $1525-1548$.

Akay A., Wickert J., Xu Z. (2000) 'Investigation of mode lock-in and friction interface', Final Report, Department of Mechanical Engineering, Carnegie Mellon University.

Allgaier R., Gaul L., Keiper W., Willnery K., Hoffmann N. (2002) 'A study on brake squeal using a beam on disc', Proceedings IMAC XX.

Baillet L., Sassi T. (2002) 'Finite element method with Lagrange multipliers for contact problems with friction', Comptes Rendus de l'Académie des Sciences Paris, Series I, Vol. 334, pp. 917-922.

Baillet L., D'Errico S., Laulagnet B. (2006) 'Understanding of the squealing noise using the temporal finite element method', Journal of Sound and Vibration, Vol. 292, pp. 443-460.

Cao Q., Ouyang H., Friswell M. I., and Mottershead J. E. (2004) 'Linear eigenvalue analysis of the disk-brake squeal problem', Int. J. for Numerical Methods in Engineering, Vol. 61, pp. 15461563.

Carpenter N.J., Taylor R.L., Katona M.G. (1991) 'Lagrange constraints for transient finite element surface contact', International Journal for Numerical Methods in Engineering, Vol. 32, pp. 130128.

Chen G.X., Zhou Z., Kapsa P., Vincent L. (2003) 'Experimental investigation into squeal under reciprocating sliding', Tribology, Vol. 36, pp. 961-971.

Culla A., Massi F. (2007) 'Introduction of uncertanties on the parametrical analysis for stability prediction' 1st International Conference on Uncertainty in Structural Dynamics, USD-2007, Sheffield, UK.

D'Ambrogio W., Sestieri A. (2001) 'Coupling theoretical data and translational FRFs to perform distributed structural modification', Mechanical Systems and Signal Processing, Vol. 15(1), pp. $157-172$.

Duffour P., Woodhouse J. (2004) 'Instability of systems with a frictional point contact. Part 1: basic modelling', Journal of Sound and Vibration, Vol. 271, pp. 365-390.

Giannini O., Akay A., Massi F. (2006) 'Experimental analysis of brake squeal noise on a laboratory brake set-up', Journal of Sound and Vibration, Vol. 292, pp. 1-20.

Giannini O., Massi F. (2008) 'Characterization of the high-frequency squeal on a laboratory brake setup', Journal of Sound and Vibration, Vol. 310 (1-2), pp. 394-408.

Fritz G., Sinou J. J., Dufal J.M., Jèzèquel L. (2007) 'Effects of damping on brake squeal coalescence patterns- application on a finite element model', Mechanics Research Communications, Vol. 34, pp. 181-190.

Kinkaid N. M., O'Reilly O. M., Papadopoulos P. (2003) 'Automotive disk brake squeal', Journal of Sound and Vibration, Vol. 267, pp. 105-166.

Massi F., Giannini O. (2005) 'Extension of a modal instability theory to real brake systems' Proc. International Modal Analysis Conference - IMAC-XXIII, Orlando, Florida.

Massi F., Giannini O., Baillet L. (2006a) 'Brake squeal as dynamic instability: an experimental investiation', Journal of the Acoustical Society of America, Vol. 120 (3), pp. 1388-1399.

Massi F. (2006b) 'Dynamic and tribological analysis of brake squeal', $\mathrm{PhD}$ thesis, University of Rome 'La Sapienza' - INSA of Lyon.

Massi F., Baillet L., Giannini O. (2006c) 'Squeal prediction on a simplified brake system by complex eigenvalues analysis', International Conference on Noise and Vibration Engineering ISMA2006, Leuven, Belgium.

Massi F., Baillet L., Giannini O., Sestieri A. (2007) 'Brake squeal phenomenon: linear and non-linear numerical approach', Mechanical Systems and Signal Processing, Vol. 21 (6), pp. 2374-2393.

Massi F., Berthier Y., Baillet L. (2008a) 'Contact surface topography and system dynamics of brake squeal', Wear, in press, available online, doi:10.1016/j.wear.2008.04.049

Massi F., Giannini O. (2008b) 'Effect of damping on the propensity of squeal instability: an experimental investigation', Journal of the Acoustical Society of America, Vol. 123(4), pp. 2017-23.

Mills H.R. (1938) 'Brake squeak', Technical Report 9000 B, Institution of Automobile Engineers.

Mottershead J.E. (1998) 'Vibration and friction-induced instability in disks', Shock and Vibration Digest, Vol. 30 (1), pp. 14-31. 
North M.R. (1972) 'Disc brake squeal, a theoretical model', Technical Report 1972/5, Motor Industry Research Association, Warwickshire, England.

Ouyang H., Nack W., Yuan Y. and Chen F. (2005) 'Numerical analysis of automotive disk brake squeal: a review', Int. J. Vehicle Noise and Vibration, Vol. 1 (3/4), pp. 207-231.

Rivin E. I. and D'Ambrogio W. (1990) 'Enhancement of dynamic quality of a machine tool using frequency response optimization method', Mechanical Systems and Signal Processing, Vol. 4, pp. $495-514$.

Sestieri A. and D'Ambrogio W. (1989) 'A modification method for vibration control of structures', Mechanical Systems and Signal Processing, Vol. 3, pp. 229-253.

Spurr R.T. (1961) 'A theory of brake squeal', Proceedings of the Automobile Division, Institution of Mechanical Engineers, 1961-1962 (1), pp. 33-52.

Tuchinda A., Hoffmann N. P., Ewins D. J. and Keiper W. (2001) 'Mode Lock-in Characteristics and Instability Study of the Pin-On-Disc System', Proceedings IMAC XIX. 


\begin{tabular}{c|cc||c|cc} 
MODE & \multicolumn{2}{|c||}{ FREQUENCY RANGE } & MODE & \multicolumn{2}{|c}{ FREQUENCY RANGE } \\
\hline \hline II support & 1200 & 2100 & $(0,4+)$ & 3141 & 3259 \\
\hline III support & 2400 & 3350 & $(0,5-)$ & 4720 & 4741 \\
\hline I pad & 3750 & 4500 & $(0,5+)$ & 4720 & 4802 \\
\hline II pad & 7300 & 12100 & $(0,6-)$ & 6600 & 6606 \\
\hline$(0,2-)$ & 1071 & 1071 & $(0,6+)$ & 6600 & 6635 \\
\hline$(0,2+)$ & 1071 & 11321 & $(0,7-)$ & 8700 & 8700 \\
\hline$(0,3-)$ & 1900 & 1900 & $(0,7+)$ & 8700 & 8730 \\
\hline$(0,3+)$ & 1900 & 2120 & $(0,8-)$ & 11000 & 11000 \\
\hline$(0,4-)$ & 3141 & 3147 & $(0,8+)$ & 11000 & 11020 \\
\hline
\end{tabular}

Table 1 - Ranges of frequency covered by the system modes. Frequency range corresponds to the variation of the normal load. 


\begin{tabular}{ccc}
\hline MASS [g] & $\begin{array}{c}\text { FREQUENCY } \\
\text { EXTREMES }[\mathrm{Hz}]\end{array}$ & $\begin{array}{c}\text { FREQUENCY } \\
\text { RANGE }[\mathrm{Hz}]\end{array}$ \\
\hline 0 & $8730-8730$ & 0 \\
2,5 & $8730-8740$ & 10 \\
5 & $8730-8745$ & 15 \\
10 & $8730-8760$ & 30 \\
\hline
\end{tabular}

Table 2: Frequency ranges covered by the $(0,7+)$ mode. 


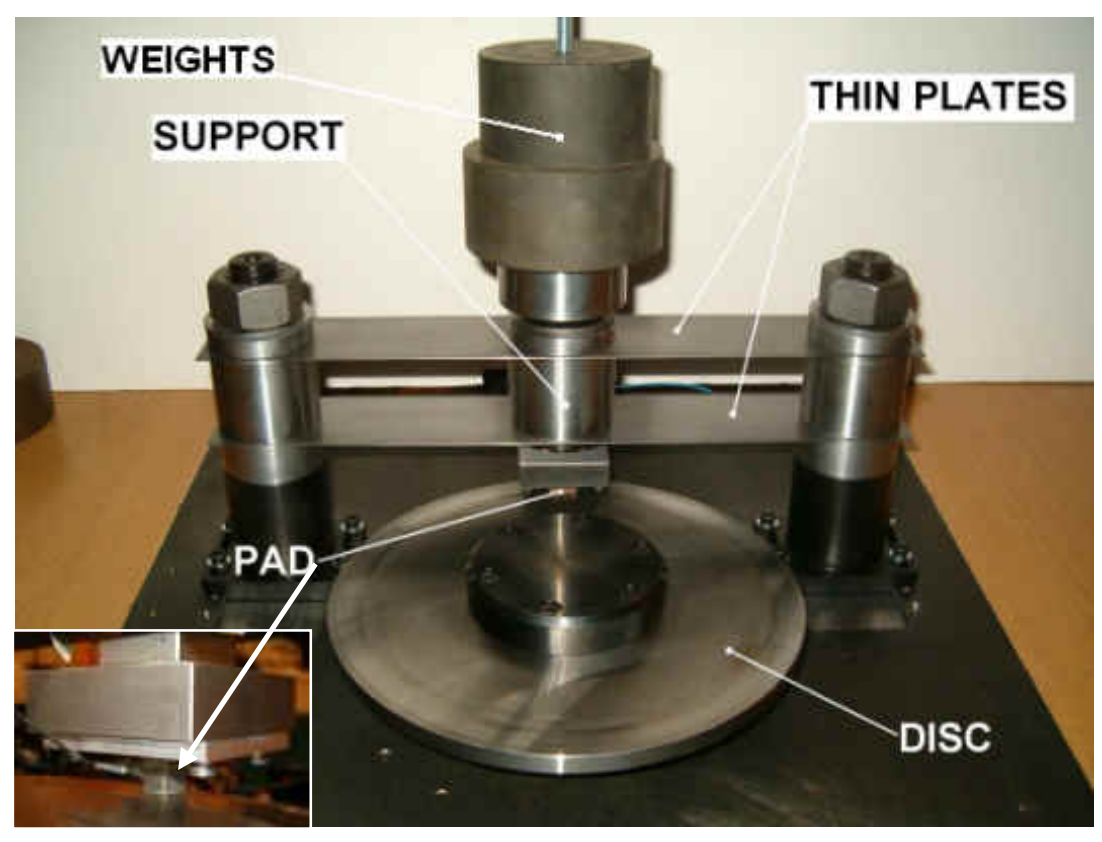

Figure 1 - Experimental set-up TriBobrake COLRIS. 


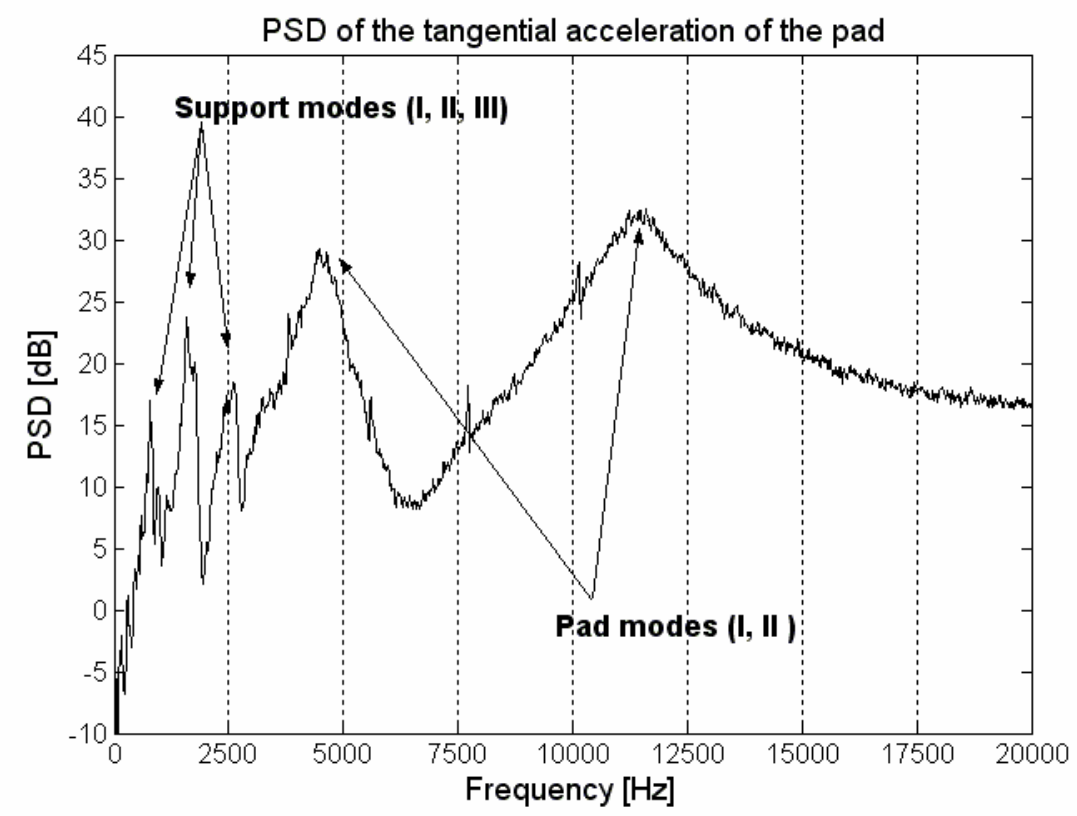

Figure 2 - PSD of the tangential acceleration of the pad during braking phase without squeal. 


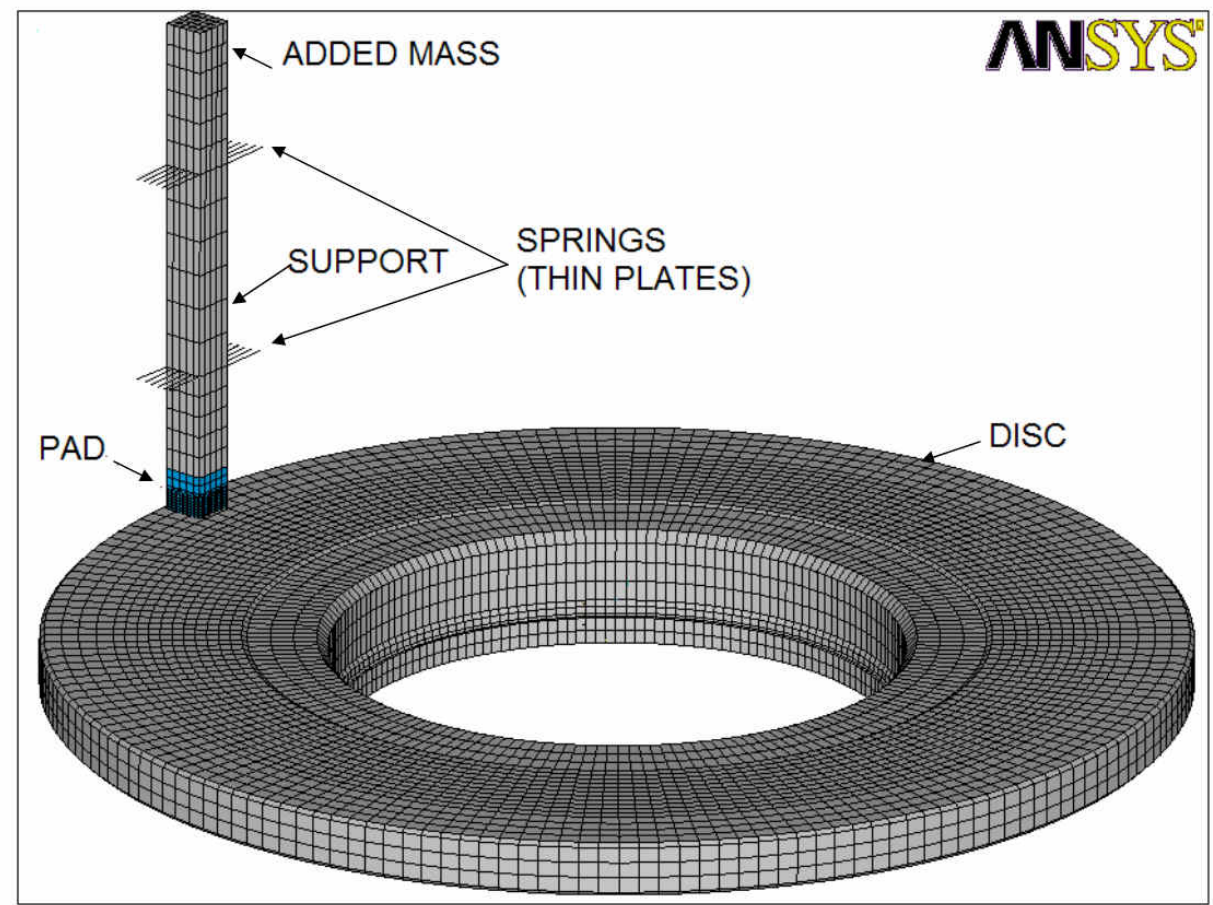

Figure 3. Finite element model of the experimental set-up. 

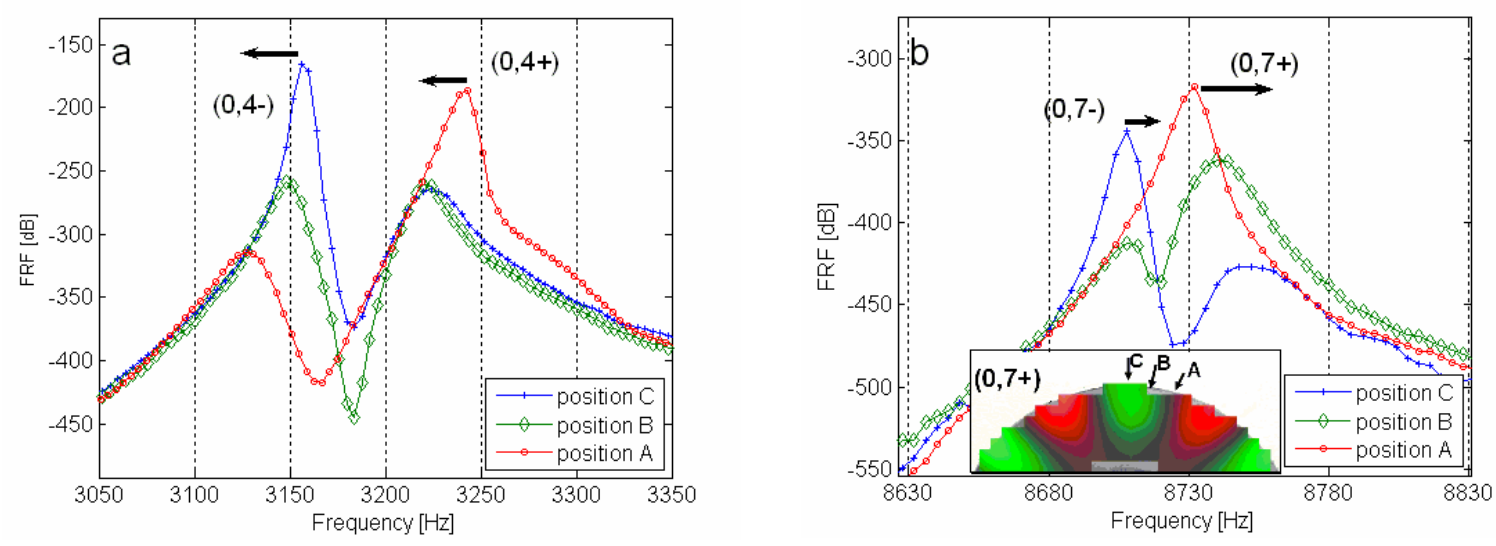

Figure 4: FRFs for different positions of the mass $(C$ is the anti-node of vibration of the modes $(n, m+)$, A its node of vibration); the arrows indicate the shift of the mode frequencies when the mass passes from position $A$ to position $C$. 

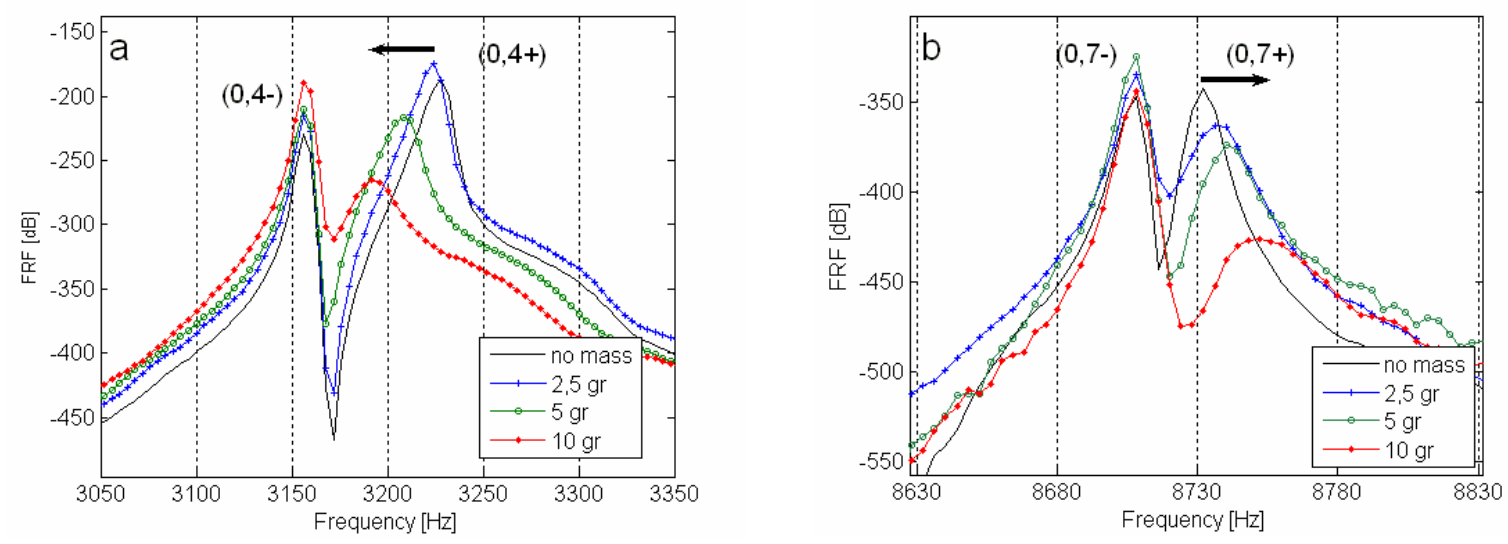

Figure 5: FRFs for different values of the mass in position $C$ (anti-node of vibration of modes $(n, m+)$; the arrows indicate the shift of mode frequencies with increasing mass. 

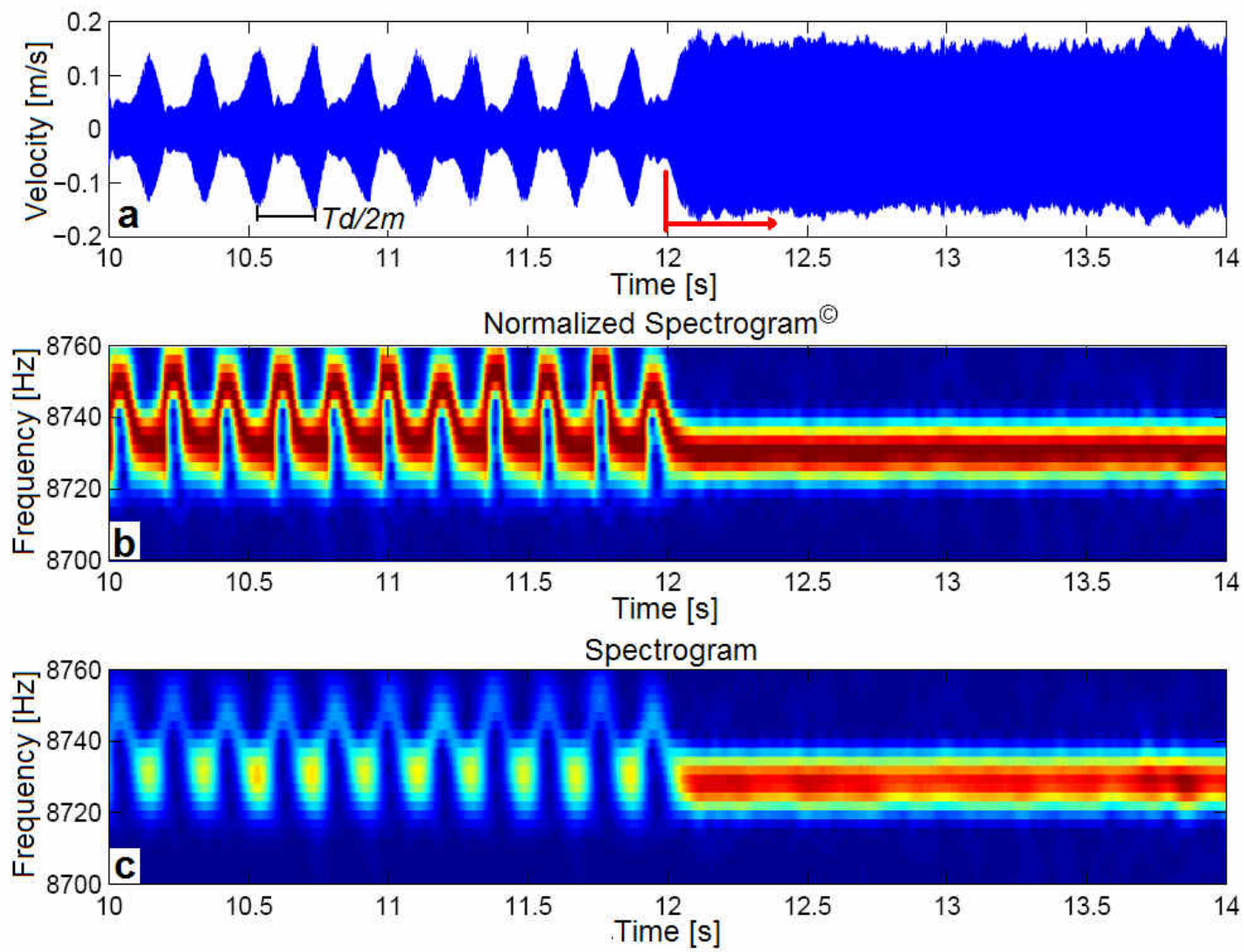

Figure 6: Tangential velocity of the pad (a), normalized spectrogram (b) and classical spectrogram (c) when a mass of $5 \mathrm{gr}$ is attached at the disc periphery; the mass is removed suddenly at $12 \mathrm{~s}$ and the squeal vibrations increase again. Unstable coupling between the II pad mode and the mode $(0,7+)$ with disc velocity of $15 \mathrm{rpm}$. Td is the period of rotation of the disc. 


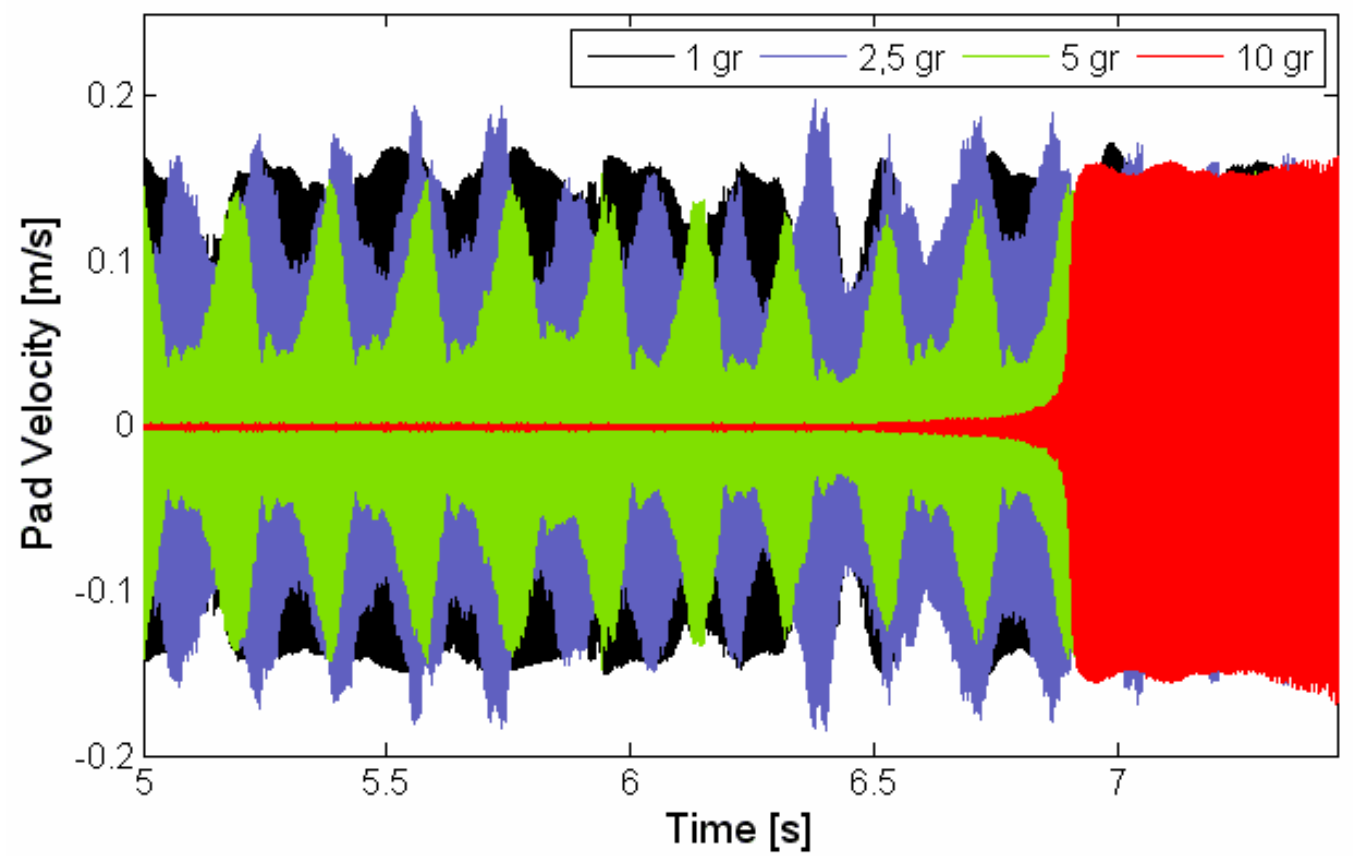

Figure 7: Tangential velocity of the pad when 1gr (black), 2.5gr (blue), 5gr (green) and 10gr are attached to the disc periphery. The mass is detached after 6.5 seconds for the measurement with 10gr. Unstable coupling between the II pad mode and the mode $(0,7+)$. 

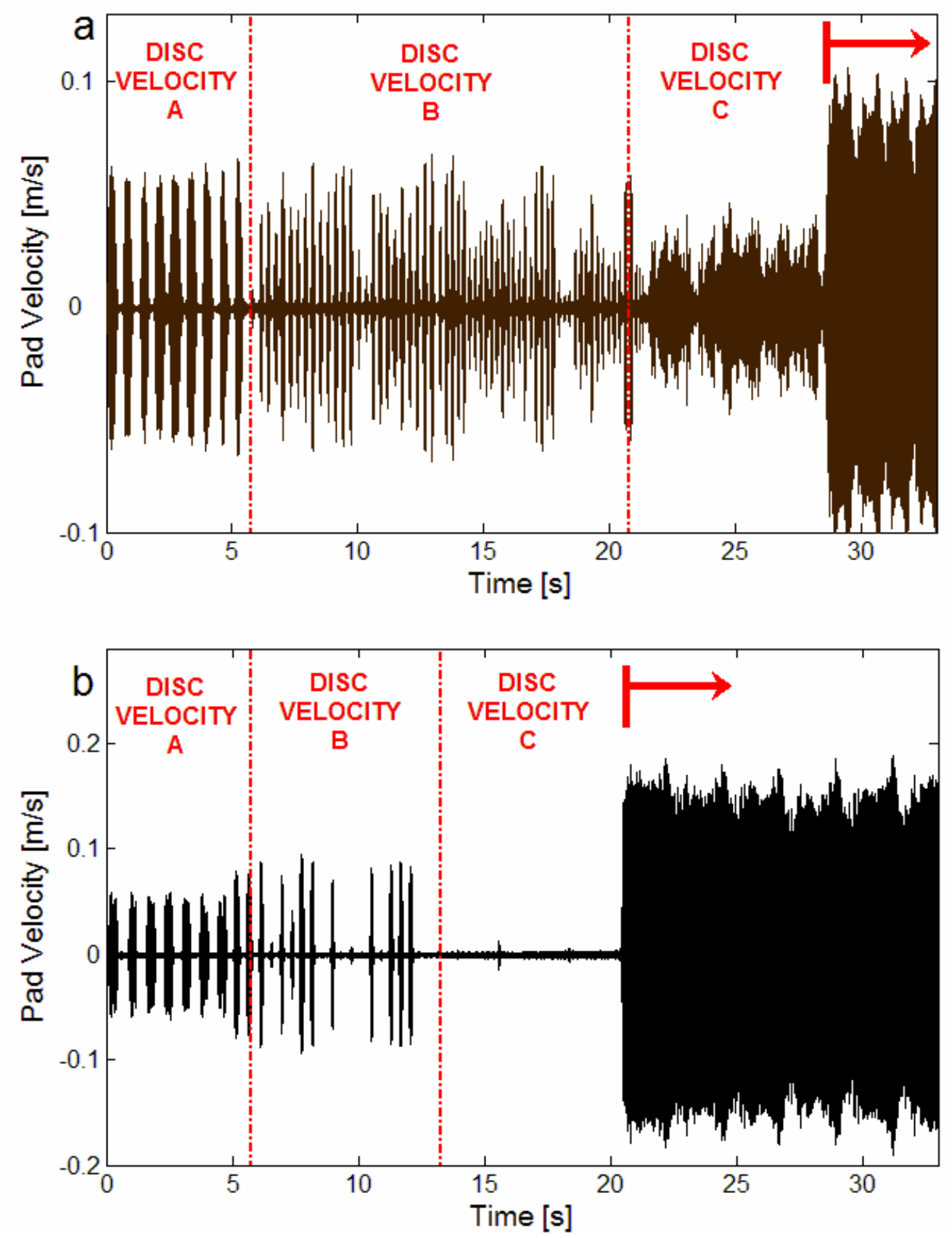

Figure 8: Tangential pad velocity for three different values of the disc rotational velocity $(A=6 \mathrm{rpm}, \mathrm{B}=12 \mathrm{rpm} ; \mathrm{C}=50 \mathrm{rpm})$, when masses of $5 \mathrm{gr}$ (a) and $7.5 \mathrm{gr}$ (b) are added to the disc periphery. The arrows indicate when the mass is detached. 


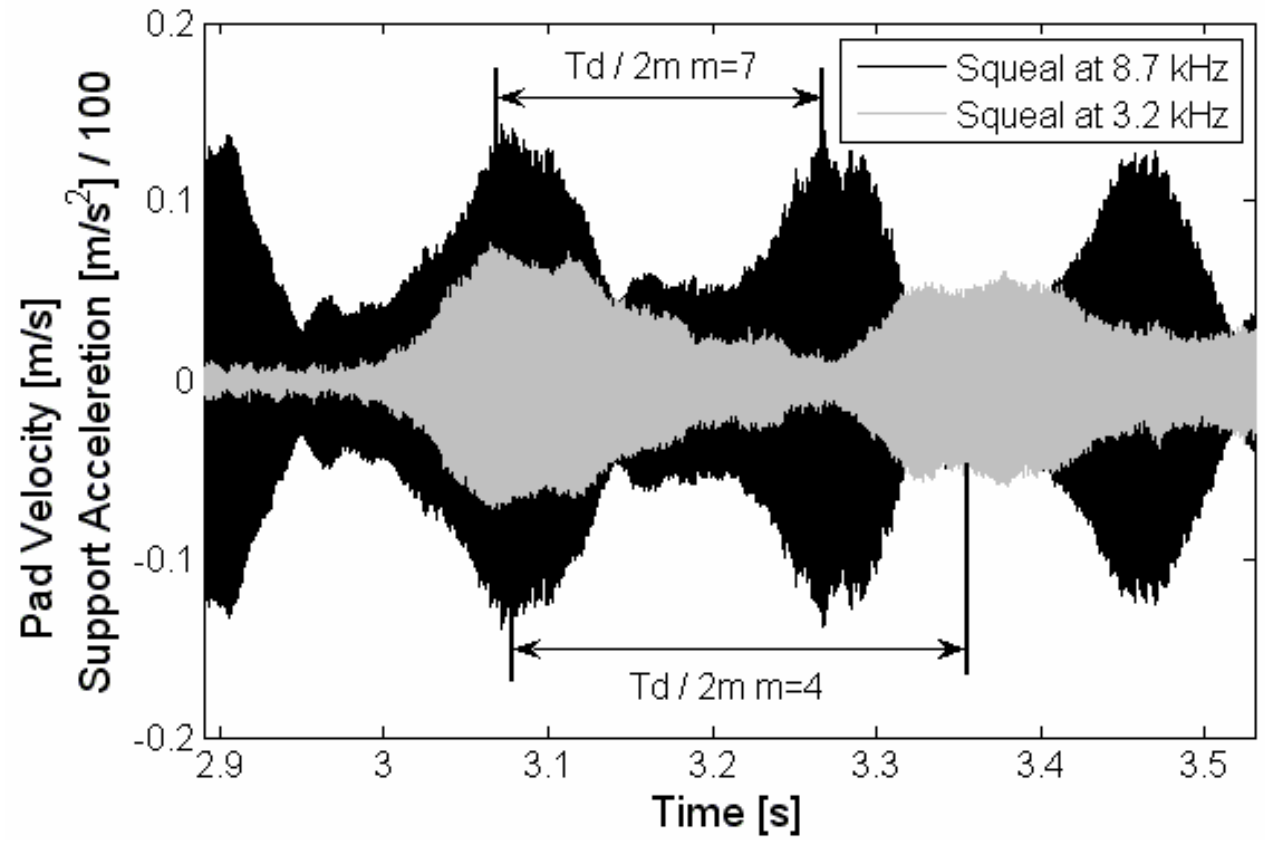

Figure 9: Pad velocity for squeal at $8.7 \mathrm{kHz}$ and $5 \mathrm{gr}$ of added mass (black); support tangential acceleration (scaled) for squeal at $3.25 \mathrm{kHz}$ and $5 \mathrm{gr}$ of added mass. 

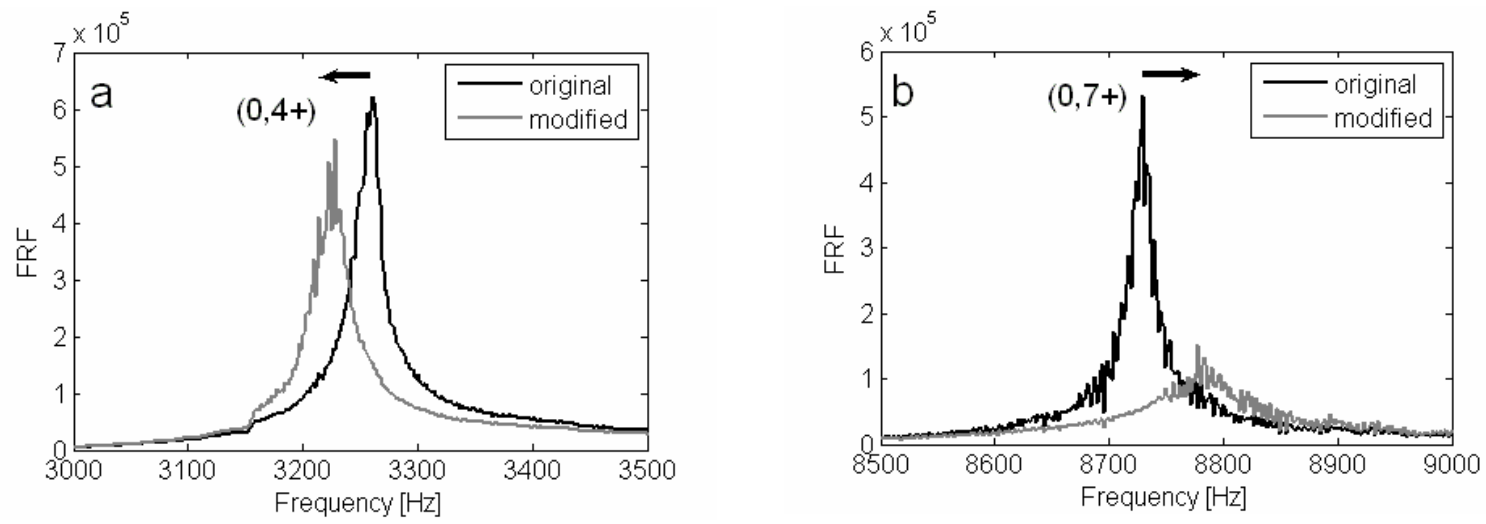

Figure 10: Comparison between the FRFs of the original system (experimental) and the modified structure by the addition of a lumped mass-damping-spring modification (mass of 10gr). The modification is introduced at the anti-node of modes $(n, m+)$. 


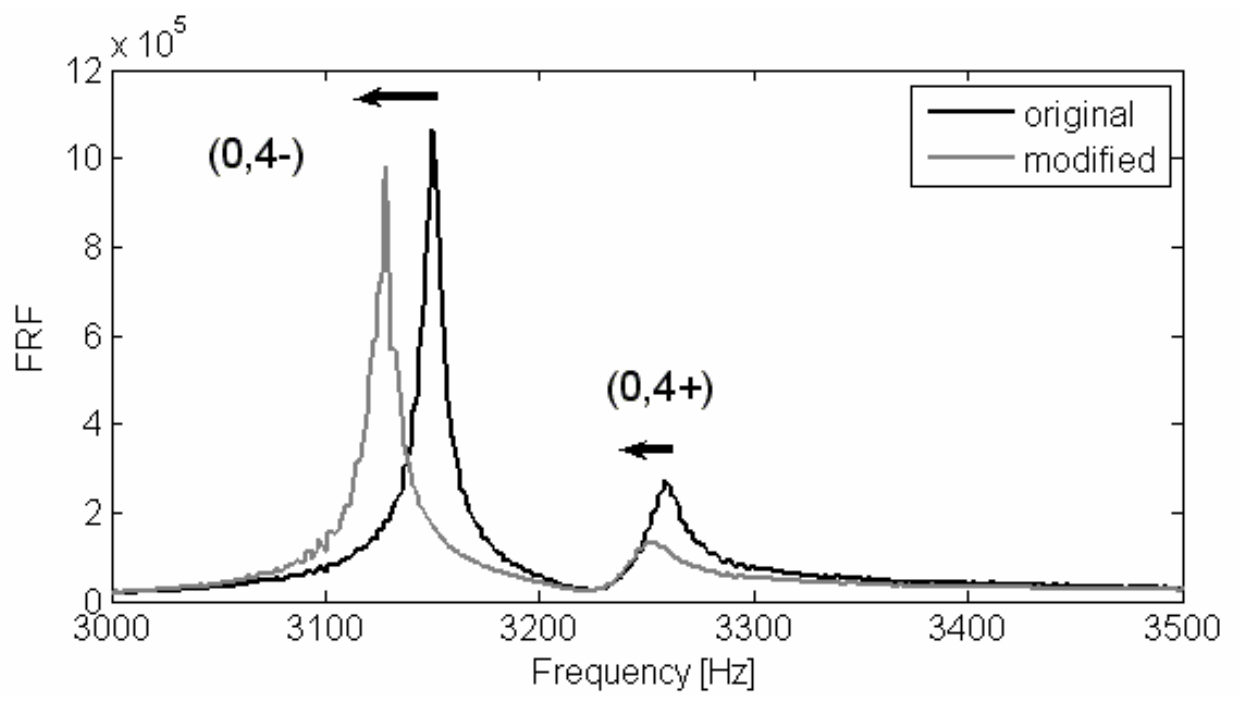

Figure 11: Comparison between the FRFs of the original system (experimental) and the modified structure by the introduction of the lumped mass-damping-spring modification (mass of 10gr) at position $B$ of mode $(0,4)$. 

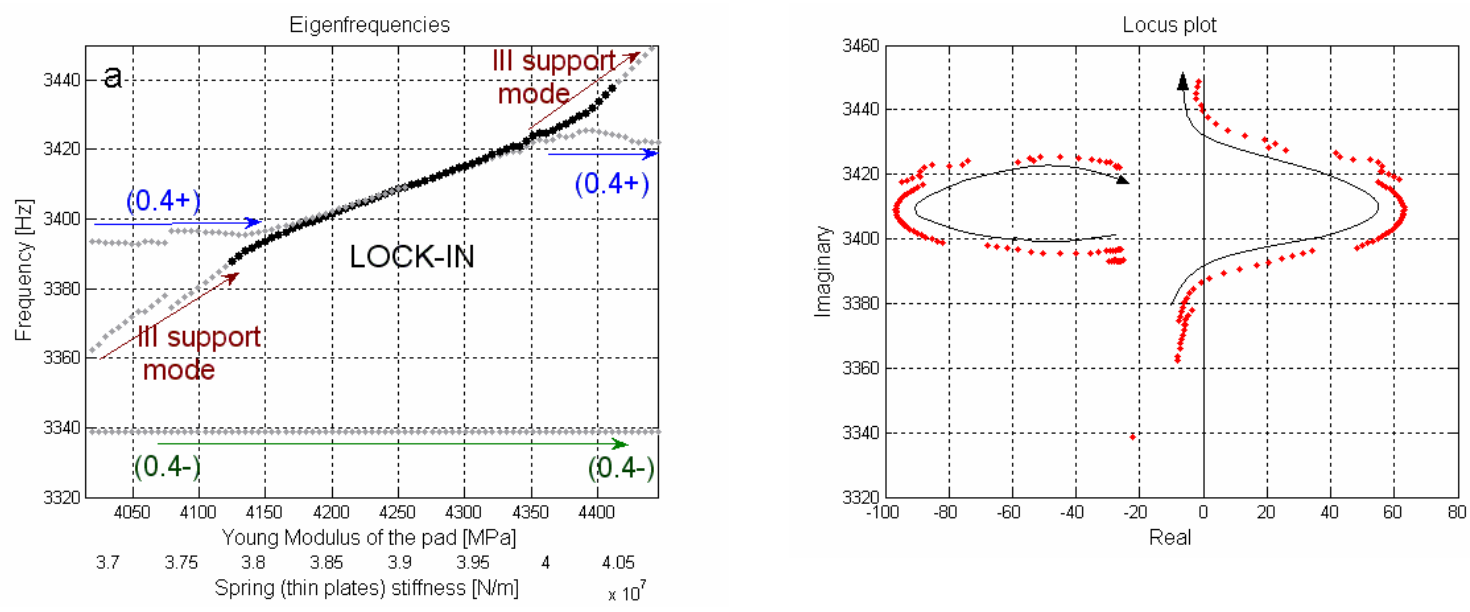

Figure 12: Lock-in between mode $(0,4+)$ and the mode of the support: a) eigenvalues as a function of the system parameters; the black dot are the eigenvalues with a positive real part (unstable); b) in the locus plot one of the two modes reaches the positive real semi-plane and becomes unstable. 

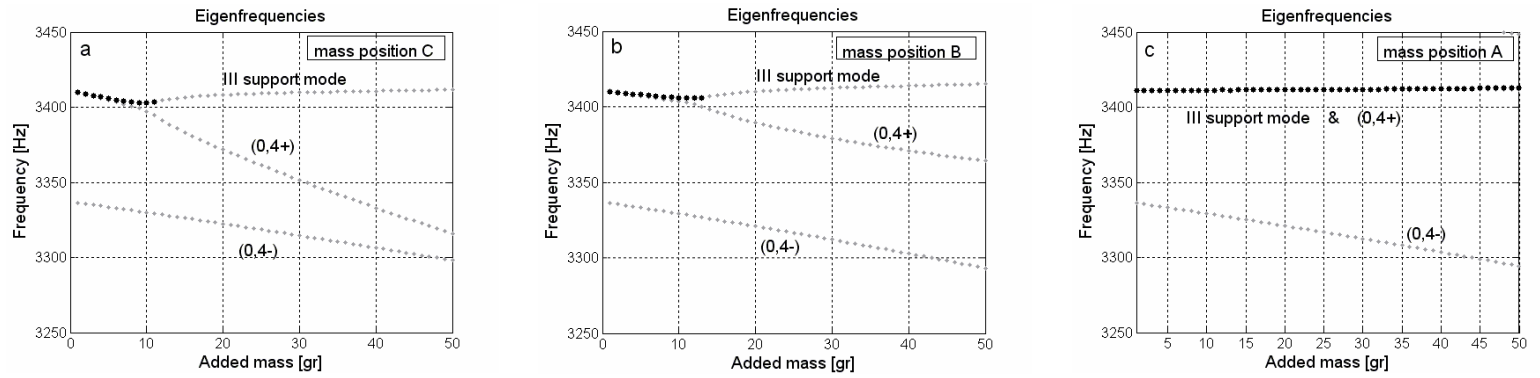

Figure 13: Effect of the addition of mass on the eigenvalues when the system parameters are set to obtain squeal without mass: a) when the mass is added in position $\mathrm{C}$ (anti-node of mode $(0,4+))$; b) the mass is added in position $B$; $c$ ) the mass is added in position $\mathrm{A}$. In this last case squeal is not affected because the mass is at the node of mode $(0,4+)$. 

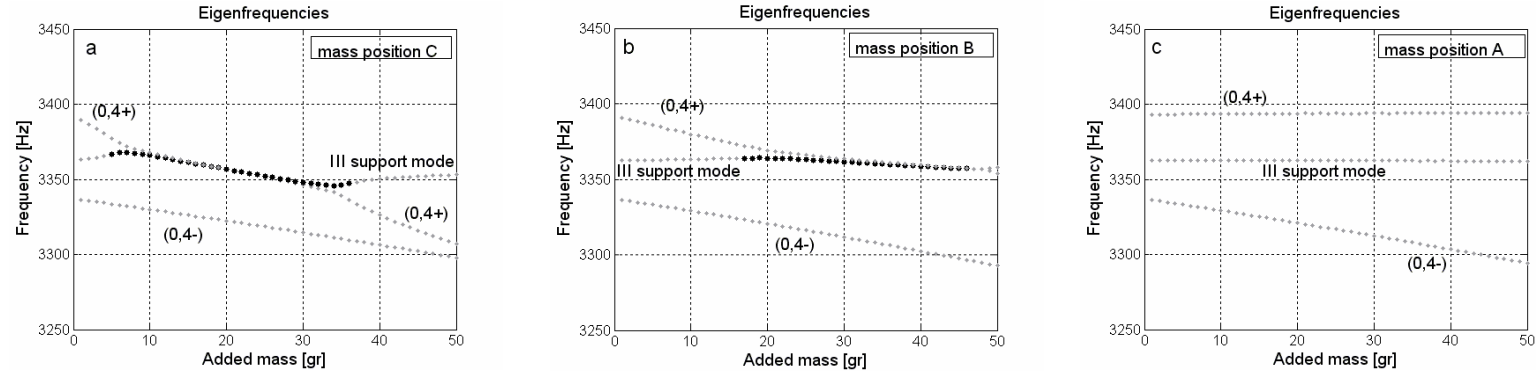

Figure 14: Effect of the addition of mass on the eigenvalues when the system parameters are set to obtain no squeal without mass (First solution on the $x$ axis of Figure 12): a) the mass is added in position $\mathrm{C}$; b) the mass is added in position $B$; $c$ ) the mass is added in position $A$. 